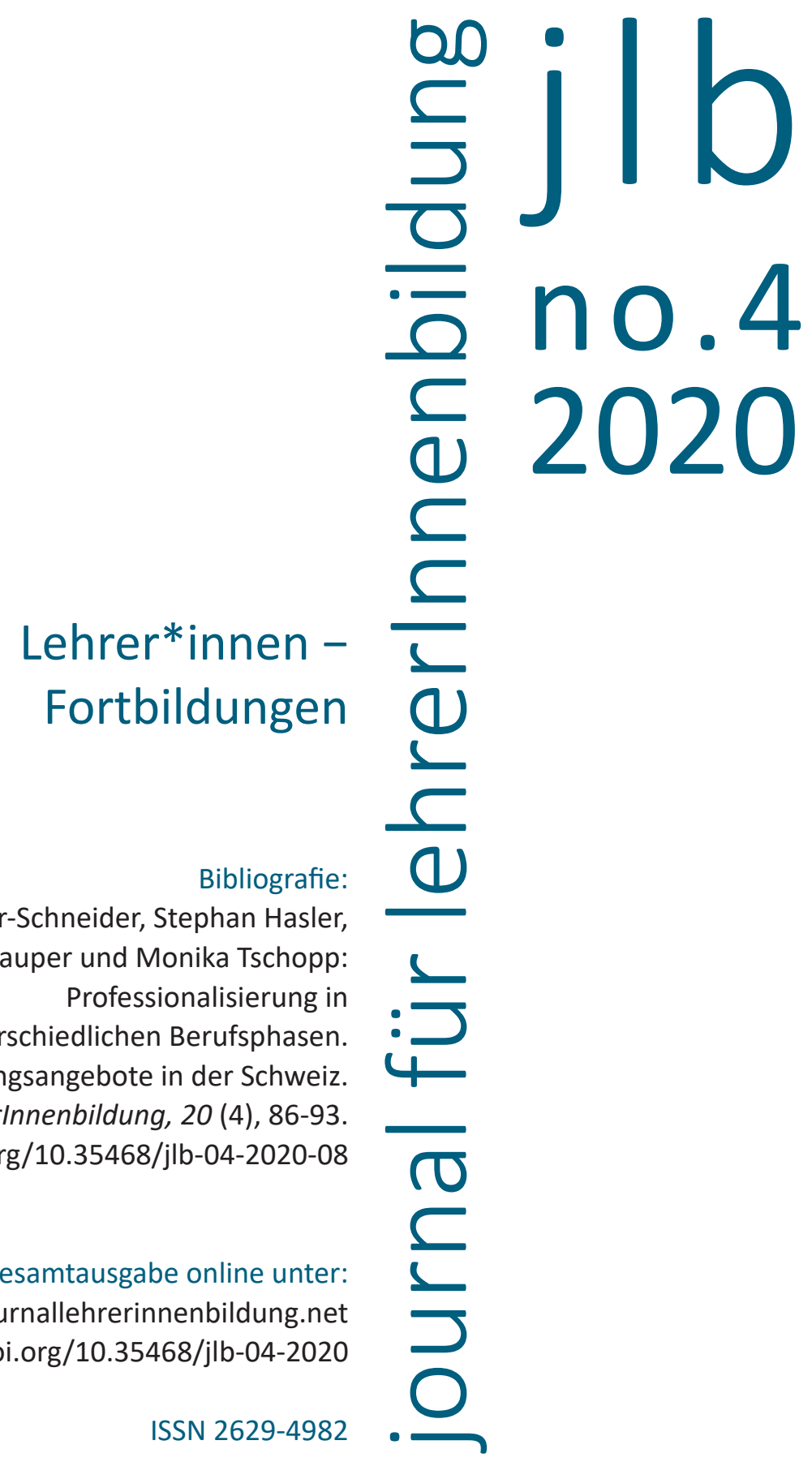


08

Manuela Keller-Schneider, Stephan Hasler, Dorothee Lauper und Monika Tschopp

\section{Professionalisierung in unterschiedlichen Berufsphasen. Weiterbildungsangebote in der Schweiz}




\section{Weiterbildung als Anforderungen an Lehrpersonen}

Professionalisierungsanforderungen erstrecken sich aufgrund von sich verändernden gesellschaftlichen und schulischen Erwartungen an die Schule und die Lehrpersonen sowie von sich verändernden individuellen Zielen der Lehrpersonen (Herzog, Sandmeier \& Terhart, in Vorb.) über die gesamte Berufsbiografie. Weiterbildung zur Unterstützung der über die Ausbildung hinausgehenden Professionalisierung ist erforderlich, damit Lehrpersonen ihre Professionalität aufgrund der laufend sich verändernden beruflichen Anforderungen erhalten und weiter ausdifferenzieren (Hericks, Keller-Schneider \& Bonnet, 2019). Der folgende Beitrag zeigt auf, wie sich die Weiterbildung in der Schweiz, die über Jahrzehnte gewachsen ist und sich laufend ausdifferenziert hat, in berufsbiografisch zeitlicher und aufgabendifferenzierend inhaltlicher Perspektive fassen lässt.

\section{Institutionelle Entwicklung von Weiterbildung}

In der Schweiz wurde die Weiterbildung für Lehrpersonen bereits im Zuge der Gründung von Seminarien zur Ausbildung von Lehrpersonen zu Beginn des 19. Jh. mitgedacht, denn die Professionalität von Lehrpersonen wurde bereits in dieser Zeit thematisiert (Bloch Pfister, 2007). Im von der Erziehungsdirektorenkonferenz (EDK) beauftragten Bericht zur Neukonzeptionalisierung der Lehrerinnen- und Lehrerbildung wurde diese in vier Phasen gegliedert über die gesamte Berufstätigkeit betrachtet (LEMO, Lehrerbildung von morgen: Müller, 1975). Unterschieden wurde zwischen einer Grundausbildung, einer Berufseinführung, einer Fortbildung zur Ergänzung und Vertiefung der Grundausbildung und einer Weiterbildung zum Erwerb zusätzlicher Qualifikationen (EDK, 1991). 1998 wurde die Differenzierung zwischen Fort- und Weiterbildung aufgehoben, Angebote in dieser Berufsphase werden seitdem als Weiterbildung bezeichnet.

Im neu gestalteten Berufsauftrag (Kull, 2015) erscheint die Weiterbildung als festgelegter Bestandteil der Jahresarbeitszeit und legt individuelle und schulinterne Weiterbildung als explizit ausgeschilderten Teil des Berufsauftrags fest. Den Pädagogischen Hochschulen wurde 
die Aufgabe der Weiterbildung von Lehrpersonen übertragen, ergänzend zu den Aufträgen Ausbildung, Forschung und Dienstleitung.

\section{Weiterbildung in unterschiedlichen Berufsphasen}

Weiterbildung setzt an der individuellen und kollektiven Ebene an und lässt sich in zeitlicher und inhaltlicher Dimension ausdifferenzieren (siehe Abb. 1). Als schulinterne Weiterbildungen auf kollektiver Ebene werden schulübergreifende Weiterbildungen zur Umsetzung der von den Volksschulämtern initiierten Reformen angeboten sowie auf die Einzelschule und ihr Schulprogramm ausgerichtete spezifische Angebote. Weiterbildung auf individueller Ebene wird bedarfs- und bedürfnisspezifisch mit der Schulleitung geplant und vereinbart.

In zeitlicher Dimension schließt an die Grundausbildung die Phase der Berufseinführung an, die fließend in das breite Weiterbildungsangebot übergeht, das mehrheitlich in der mittleren Berufsphase genutzt wird; eine Abschlussphase schließt den Bogen der Berufsarbeit.

In inhaltlicher Dimension lassen sich Angebote der Weiterbildung in Bereiche des Berufseinstiegs und des Berufsabschlusses, sowie der Vertiefung im Kernbereich, der Profilbildung in spezifischen Bereichen, der Aktualisierung für einen Wiedereinstieg und der Spezialisierung für spezifische Funktionen gliedern. Die Phasen und ihre Angebote lassen sich wie folgt beschreiben.

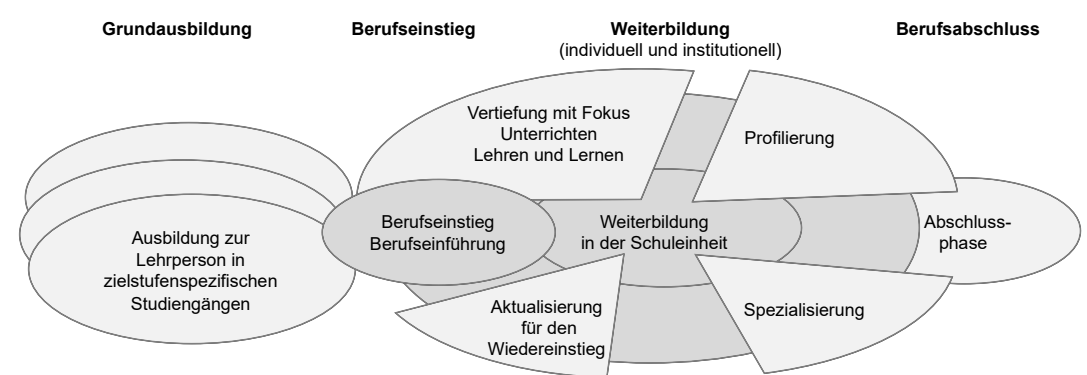

Abb. 1 Phasen in der Professionalisierung von Lehrpersonen (nach Lauper, Tschopp, Hasler \& Keller-Schneider, 2017) 


\section{Berufseinstieg und Berufseinführung}

Der Einstieg in die eigenverantwortliche Berufstätigkeit stellt aufgrund der sprunghaft ansteigenden Komplexität der Dynamik gleichzeitig zu meisternder Aufgaben Herausforderungen an alle Lehrpersonen, die berufsphasenspezifisch konkretisierten Entwicklungsaufgaben anzunehmen und zu bewältigen, um im Beruf anzukommen. Diese umfassen die Bereiche der identitätsstiftenden Rollenfindung, der adressatenbezogenen Vermittlung, der anerkennenden Klassenführung, sowie der mitgestaltenden Kooperation in und mit der Institution (Keller-Schneider, 2020). Trotz der in die Ausbildung integrierten Schulpraxis mit zunehmend anspruchsvoller gestalteten Aufgaben stellen sich in der Berufseinstiegsphase erneut Anforderungen. Diese können strukturbedingt im Rahmen einer Ausbildung (in der Schweiz einphasig mit integrierter Praxis) aufgrund der fehlenden Eigenverantwortung nicht vorweggenommen werden. Institutionelle Angebote der Berufseinführung für Lehrpersonen in der Berufseinstiegsphase werden den Berufseinsteigenden von den Pädagogischen Hochschulen angeboten. Sie fokussieren auf die berufsphasenspezifischen Anforderungen und die daraus hervorgehenden Anliegen der Lehrpersonen.

Angebote der Berufseinführung mit zunehmend erweiterten Zielen (Schneuwly, 1996) wurden seit den 1970er Jahren nach kantonalen Schwerpunktsetzungen entwickelt. Am Anfang stand insbesondere die Einfindung in den Beruf als Einsozialisation in die lokale Schule im Vordergrund, ergänzt um schulexterne Beratungs- und Unterstützungsangebote. Diese eher defizitär ausgerichteten Weiterbildungsansätze wurden um den Schwerpunkt einer berufsphasenspezifischen Weiterbildung ergänzt. Darin zeigt sich, dass die Berufseinstiegsphase als eigene, an die Grundausbildung anschließende berufsbiografische Phase mit spezifischen Anforderungen und darauf bezogenem Weiterbildungsbedarf anerkannt wurde. Seit der Gründung der Pädagogischen Hochschulen in der Schweiz (ab 2002) kam die Zielsetzung hinzu, über die Bearbeitung von subjektiv erfahrenen Herausforderungen die Lehrpersonen in ihrer Professionalisierung und damit ihr über den Berufseinstieg hinausgehendes Professionalisierungsbewusstsein zu fördern. Aktuell umfassen diese Angebote eine kollegiale Begleitung durch Lehrpersonen derselben Schule, reflexionsorientiere Supervision sowie an die Erfahrung anknüpfende Weiterbildung (Keller-Schneider, 2019). 


\section{Weiterbildung \\ mit unterschiedlichen Fokussierungen}

Individuelle Weiterbildungen der mittleren Berufsphase umfassen vielfältige Angebote. Die Lehrperson kann im Kontext ihrer Schule ihre Weiterbildung und damit auch die Berufsphase und Berufslaufbahn different gestalten.

- Angebote der Vertiefung und Erweiterung von auf Unterricht- und Lehr-/Lernprozesse bezogenen Kompetenzen und Interessen fördern die Lehrperson in ihrer Kernaufgabe und erweitern durch Impulse die Palette von Erprobtem und Bewährtem. Diese Angebote umfassen Themen wie schülerorientierte Unterrichtskonzepte, Beurteilungspraxis, kooperatives Lernen, partizipatives Unterrichten, Beziehungsgestaltung, Zusammenarbeit, Konfliktbewältigung, Kompetenzorientierung im Unterricht, Selbststeuerungsfähigkeiten, soziales Lernen, sowie fachliche und fachdidaktisch spezifizierte Angebote.

- Angebote zur Profilierung ermöglichen, die Kernaufgabe als Lehrperson durch eine Vertiefung eines spezifischen Bereichs zu erweitern und damit ein individuell geprägtes Profil berufsrelevanter Kompetenzen zu entwickeln. Über eine Profilbildung ist auch die Übernahme von auf schulebene relevanten Aufgaben möglich. Profilbildende Weiterbildungen umfassen Bereiche wie die Weiterbildung zur Praxislehrperson, zur Begleitung von Berufseinsteigenden, zum IT-Spezialisten bzw. zur IT-Spezialistin, in Theaterpädagogik, Schülerpartizipation oder Begabungs- und Begabtenförderung.

- Angebote zur Aktualisierung richten sich an Lehrpersonen, die nach einigen Jahren des Unterbruchs ihrer Lehrtätigkeit einen Wiedereinstieg in den Beruf erwägen oder bereits eingestiegen sind. Thematisiert werden insbesondere aktuelle Lehrmittel und didaktische Konzeptionen, sowie Neuerungen im Schulfeld und damit verbundene Veränderungen der Aufgaben und der Rolle einer Lehrperson.

- Angebote der Spezialisierung umfassen Weiterbildungen, die über die Kernaufgabe von Lehrpersonen hinausführen und zur Übernahme von Spezialaufgaben in einer Schule führen, wie Deutsch als Zweitsprache (DaZ), schulische Heilpädagogik oder Schulleitung. Die Weiterbildung zur Schulleitung unterscheidet sich von den an- 
deren Spezialisierungen darin, dass die Schulleitungsarbeit in eine dem Kollegium übergeordnete Rolle und damit zu einer anderen

Stellung im schulischen Gefüge führt und in der Regel mit einem Schulwechsel verbunden wird. ${ }^{1}$

\section{Berufsabschlussphase}

Berufsbezogene Weiterbildungen in der Phase des Berufsabschlusses sind erst wenig ausdifferenziert, zu berufsübergreifenden Pensionierungsvorbereitungskursen werden Lehrpersonen zusammen mit kantonal Abgestellten anderer Berufsgruppen rund fünf Jahre vor der regulären Pensionierung eingeladen.

Auf den Lehrberuf bezogene berufsphasenspezifische Weiterbildungen zum Abschluss der beruflichen Tätigkeit als Lehrpersonen fokussieren insbesondere die individuelle Ebene. Thematisiert wird, was die Lehrperson in der letzten Berufsphase noch umsetzen und erreichen oder was sie weitergeben möchte und welche Aufgaben in dieser Phase nicht mehr übernommen, $d$. h. anderen überlassen und zugetraut werden. Über die Weiterbildung hinausgehend ist als Aufgabe der Schulleitung von Bedeutung, zur Umsetzung dieser Pläne und Wünsche beizutragen, zu Erhaltendes zu sichern und auch spezifische Erwartungen zu formulieren, denen sich berufsabschließende Lehrpersonen stellen sollen.

\section{Abschluss}

Parallel zur Ausdifferenzierung von Weiterbildungsmöglichkeiten über die gesamte Berufslaufbahn hinweg wurde Weiterbildung als Teil der Berufsarbeit im Berufsauftrag von Lehrpersonen verankert. Damit umfasst in der Schweiz die von der Profession und ihrem Dachverband postulierte Weiterbildungspflicht $(\mathrm{LCH}, 2008)$ einen anerkannten und verbindlichen Anteil der Berufsarbeit.

1 Da in der Schweiz Anstellungen von Lehrpersonen gegenseitig kündbar sind (keine Verbeamtung), sucht sich eine in die Rolle der Schulleitung wechselnde Lehrperson in der Regel eine Anstellung an einer anderen Schule. 


\section{Literatur}

Bloch Pfister, A. (2007). Priester der Volksbildung. Zürich: Chronos.

EDK (1991). Lehrerfortbildung von morgen (Dossier 16A). Bern: EDK.

Hericks, U., Keller-Schneider, M. \& Bonnet, A. (2019). Professionalität von Lehrerinnen und Lehrern in berufsbiographischer Perspektive. In M. Harring, C. Rohlfs \& M. Gläser-Zikuda (Hrsg.), Handbuch Schulpädagogik (S. 597-607). Münster: Waxmann utb.

Herzog, A., Sandmeier, A. \& Terhart, E. (in Vorb.). Berufliche Biografien und berufliche Mobilität von Lehrerinnen und Lehrern. In T. Hascher, W. Helsper \& T. S. Idel (Hrsg.), Handbuch Schulforschung. Wiesbaden: Springer.

Keller-Schneider, M. (2019). Professionalisierung im Berufseinstieg von Lehrpersonen. In M. Syring \& S. Weiß (Hrsg.), Lehrer/in sein - Lehrer/in werden - die Profession professionalisieren (S. 145-160). Bad Heilbrunn: Klinkhardt.

Keller-Schneider, M. (2020). Entwicklungsaufgaben im Berufseinstieg von Lehrpersonen. Bearbeitung beruflicher Herausforderungen im Zusammenhang mit Kontext- und Persönlichkeitsmerkmalen sowie in berufsphasendifferenten Vergleichen (2., überarbeitete und erweiterte Aufl.). Münster: Waxmann.

Kull, M. (2015). Berufsauftrag. Zürich: Bildungsdirektion, Volksschulamt.

Lauper, D., Tschopp, M., Hasler, S. \& Keller-Schneider, M. (2017). Weiterbildung in der mittleren Berufsphase (internes Arbeitspapier). Luzern: Pädagogische Hochschule Luzern.

LCH (2008). LCH-Berufsleitbild, LCH-Standesregeln. Verfügbar unter https://www.lch. ch/fileadmin/files/documents/Verlag_LCH/LCH-Berufsleitbild_Standesregeln.pdf [19.10.2020].

Müller, F. (1975). Lehrerbildung von morgen (LEMO). Hitzkirch: Comenius-Verlag.

Schneuwly, G. (1996). Berufseinführung von Lehrerinnen und Lehrern (Dossier 40A). Bern: EDK. 
Manuela Keller-Schneider, Prof. Dr. phil., Pädagogische Hochschule Zürich.

Arbeitsschwerpunkte: Professionsforschung, insbesondere Berufseinstieg, Team und Schulentwicklung, selbstreguliertes Lernen

m.keller-schneider@phzh.ch

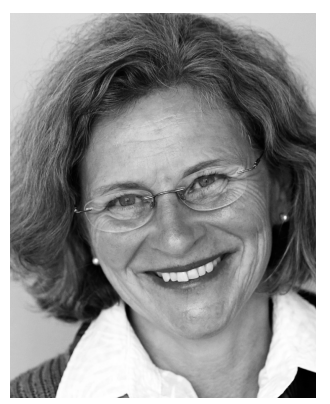

Stephan Hasler, Berater, Dozent und Fachbereichsverantwortlicher Berufseinstieg und Berufslaufbahn, PH Bern. Arbeitsschwerpunkte:

Personzentrierte Beratung, Begleitung von Berufseinsteigenden

stephan.hasler@phbern.ch

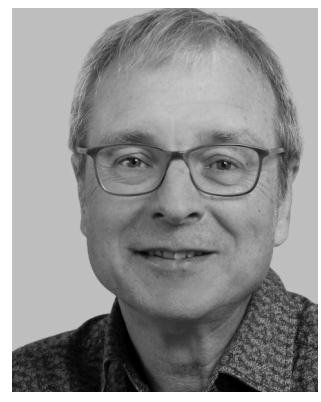

Dorothee Lauper, lic. phil., Pädagogische Hochschule Luzern. Arbeitsschwerpunkte: Berufsbiografische Angebote,

Standortbestimmungen und Weiterbildungsberatungen für Lehrpersonen

dorothee.lauper@phlu.ch

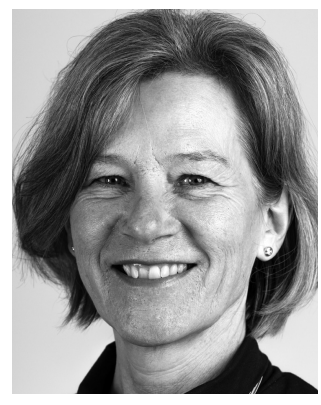

Monika Tschopp, Leiterin Ressort Berufsbiographie und Laufbahn, PH Fachhochschule Nordwestschweiz. Arbeitsschwerpunkte: Erwachsenenbildung, Personal- und Organisationsentwicklung an Schulen, Kompetenzorientierung und -entwicklung

monika.tschopp@fhnw.ch

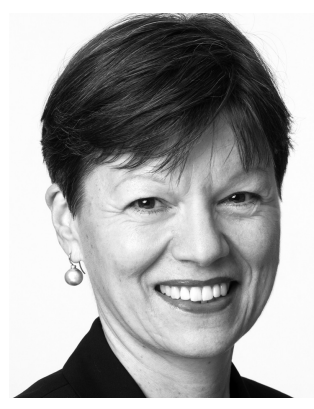

